\title{
The influence of density stratification and multiple nonlinearities on solar torsional oscillations
}

\author{
E. Covas ${ }^{1}$, D. Moss ${ }^{2}$, and R. Tavakol ${ }^{1}$ \\ 1 Astronomy Unit, School of Mathematical Sciences, Queen Mary, University of London, Mile End Road, \\ London E1 4NS, UK \\ e-mail: e.o.covas@qmul.ac.uk \\ 2 Department of Mathematics, The University, Manchester M13 9PL, UK \\ e-mail: moss@ma.man.ac.uk
}

Received 3 July 2003 / Accepted 3 December 2003

\begin{abstract}
Analyses of recent helioseismic data have produced ample evidence for substantial dynamical variation of the differential rotation within the solar convection zone. Given the inevitable difficulties in resolving the precise nature of variations in deeper layers, much effort has recently gone into determining theoretically the expected modes of behaviour, using nonlinear dynamo models. Two important limitations of these models are that they have so far included only one form of nonlinearity, and as yet they have not taken into account the density stratification in the solar convection zone. Here we address both of these issues by studying the effects of including density stratification, as well as including an $\alpha$-quenching nonlinearity in addition to the previously studied effects of the Lorentz force on the differential rotation. We find that observationally important features found in the earlier uniform density models remain qualitatively unchanged, although there are quantitative differences. This is important as it provides more realistic theoretical predictions to be compared with and guide observations, especially in the deeper regions where the uncertainties in the inversions are larger. However the presence of an effective alpha-quenching nonlinearity significantly reduces the amplitudes of the oscillations.
\end{abstract}

Key words. Sun: magnetic fields - Sun: activity - Sun: oscillations

\section{Introduction}

Recent analyses of the helioseismic data have produced a wealth of information concerning the dynamical modes of behaviour associated with the differential rotation in the solar convection zone (CZ). Previous analyses of data from the Michelson Doppler Imager (MDI) instrument on board the SOHO spacecraft (Howe et al. 2000a) and the Global Oscillation Network Group (GONG) project (Antia \& Basu 2000) have found that torsional oscillations penetrate into the convection zone, to depths of at least 10 percent in radius, but did not agree on the behaviour in the poorly resolved region near the bottom of the convection zone. In order to understand these observed dynamical phenomena theoretically, and partly motivated by these apparent discrepancies, we have previously made detailed studies of the observed variations in the differential rotation in the $\mathrm{CZ}$ in the framework of nonlinear dynamo models which included the nonlinear action of the azimuthal component of the Lorentz force of the dynamo generated magnetic field on the solar angular velocity

Send offprint requests to: $\mathrm{R}$. Tavakol, e-mail: r.tavakol@qmul.ac.uk
(Covas et al. 2000a,b, 2001a,b; Tavakol et al. 2002). These studies produced a number of important robust features, that may be treated as their predictions. These include: (i) the robust existence of torsional oscillations that penetrated all the way down to the bottom of the convection zone for near-critical and moderately supercritical dynamo regimes; (ii) the existence of parameter ranges for which the supercritical models show spatiotemporal fragmentation, with different oscillatory modes of behaviour at the top and the bottom of the CZ; (iii) the existence of equatorial as well as polar branches in the angular velocity residuals; and (iv) the presence of secondary modulations on these equatorial branches. Predictions (i) and (ii) are in principle able to account for different aspects of earlier observations. Interestingly, the most recent inversions by Vorontsov et al. (2002a) demonstrate further important agreement with our predictions. In particular they provide strong evidence that these oscillations do in fact penetrate down to the bottom of the CZ. Furthermore they find clear evidence for the presence of polar branches as well as of modulations on the equatorial branch (see Vorontsov et al. 2002b for preliminary results, and also Vorontsov et al. 2003). 
Despite these successes, there are two important issues concerning these models that require further study. Firstly, they ignore an important feature of the real Sun, namely the presence of a strong density stratification in the solar convection zone and, secondly, they involve only one form of nonlinearity - that due to the Lorentz force acting on the large-scale velocity field (here just the rotational flow). A more realistic model would be expected to include other forms of nonlinearity, such as the effects of the Lorentz force on the small-scale motions, for example as naively modelled by a nonlinear $\alpha$-quenching. Investigating the consequences of these shortcomings is particularly important, given the limitations on the length of the helioseismic data sets and the resulting uncertainties in the inversions, especially regarding the behaviour in the lower parts of the CZ (see e.g. Vorontsov et al. 2002).

The aim of this paper is to improve our previous model by (i) including a density stratification in our previous model, and (ii) by adding a second form of nonlinearity in the form of an explicit $\alpha$-quenching. In contrast, in our previous work we imposed a radial variation of alpha that we felt might be consistent with a strong quenching in the overshoot region where the magnetic field was expected to be strong (by putting $\alpha=0$ in this region). We can now make a detailed study of both the effects of density stratification and the competing forms of nonlinearity on various observationally important features of our model. Throughout the underlying zero order angular velocity is taken to be consistent with the recent inversions of the helioseismic (MDI) data.

The structure of the paper is as follows. In the next section we outline our model, and Sects. 3 and 4 contain our detailed results. In Sect. 5 we attempt to draw some conclusions.

\section{The model}

\subsection{Equations}

We write the large-scale velocity field in the form $u=v \hat{\phi}-\frac{1}{2} \nabla \eta$, and put $v=v_{0}+v^{\prime}, v_{0}=\Omega_{0} r \sin \theta$ being the underlying (assumed known) solar rotation law. Thus we solve the standard mean-field dynamo equation

$\frac{\partial \boldsymbol{B}}{\partial t}=\nabla \times(\boldsymbol{u} \times \boldsymbol{B}+\alpha \boldsymbol{B}-\eta \nabla \times \boldsymbol{B})$,

for the mean magnetic field and the corresponding equation

$$
\frac{\partial v^{\prime}}{\partial t}=\frac{(\nabla \times \boldsymbol{B}) \times \boldsymbol{B}}{4 \pi \rho r \sin \theta} \cdot \hat{\phi}+\rho^{-1} \nabla \cdot \tau,
$$

( $\tau$ the stress tensor) for the deviations from the underlying ( mean) rotational velocity $v_{0}$; as usual $\eta$ and $v$ are turbulent transport coefficients. We proceed as described in Moss \& Brooke (2000) and in previous papers in this series, Nondimensionalizing in terms of the solar radius $R$ and a time $R^{2} / \eta_{0}$, where $\eta_{0}$ is the maximum value of $\eta$, and putting $\Omega=\Omega^{*} \tilde{\Omega}, \alpha=\alpha_{0} \tilde{\alpha}, \eta=\eta_{0} \tilde{\eta}, \boldsymbol{B}=B_{0} \tilde{\boldsymbol{B}}$ and $v^{\prime}=\Omega^{*} R \tilde{v}^{\prime}$. This gives dynamo parameters $R_{\alpha}=\alpha_{0} R / \eta_{0}, R_{\omega}=\Omega^{*} R^{2} / \eta_{0}$, $P_{\mathrm{r}}=v_{0} / \eta_{0}$, where $\Omega^{*}$ is the solar surface equatorial angular velocity and $B_{0}=\left(\Lambda 4 \pi \rho_{0} \eta_{0} \Omega^{*}\right)^{1 / 2}$, where $\Lambda$ is an arbitrary scaling factor and $\rho_{0}$ is the density at the lower boundary of the model. As discussed in Moss \& Brooke (2000), the physical field $B_{0} \tilde{\boldsymbol{B}}$ is independent of the value of $\Lambda$ - for "historical" reasons we set $\Lambda=10^{4}$. $P_{\mathrm{r}}$ is the turbulent Prandtl number.

The model includes a density stratification discussed in Sect. 2.2 below, as well as a second source of nonlinearity in the form of an $\alpha$-quenching discussed in Sect. 2.3 below.

In this investigation, $\Omega_{0}$ is given in $0.64 \leq r \leq 1$ by an interpolation on the MDI data obtained from 1996 to 1999 (Howe et al. 2000a). For $\alpha$ we put $\alpha=\alpha_{\mathrm{r}}(r) f(\theta)$, where $f(\theta)$ was chosen to be $\sin ^{2} \theta \cos \theta$. This form has been used previously (see e.g. Rüdiger \& Brandenburg 1995) and its choice here is simply to make the butterfly diagrams more realistic. For $\alpha_{\mathrm{r}}$ we took the following choices. In the absence of $\alpha$-quenching, $\alpha_{\mathrm{r}}$ was set to be $\alpha_{\mathrm{r}}=1$ for $0.7 \leq r \leq 0.8$ with cubic interpolation to zero at $r=r_{0}$ and $r=1$. In presence of $\alpha$-quenching, we took $\alpha_{\mathrm{r}}=1$ throughout the convection zone. We use the convention that $\alpha_{\mathrm{r}}>0$ and $R_{\alpha}<0$, to get the correct sense of migration of field patterns. Also, in order to take into token account the likely decrease in the turbulent diffusion coefficient $\eta$ in the overshoot region, we allowed a simple linear decrease from $\tilde{\eta}=1$ at $r=0.8$ to $\tilde{\eta}=0.5$ in $r<0.7$.

At the surface $r=R$ we took vacuum boundary conditions, whereby the poloidal field within $r=R$ is smoothly joined, by a matrix multiplication, to an external vacuum solution; the azimuthal field there satisfies $B=0$. At the inner boundary $r=r_{0}$ we used the same conditions as Tavakol et al. (2002). We note that "reasonable" changes in these boundary conditions do not qualitatively change our conclusions (cf. Tavakol et al. 2002).

We set $r_{0}=0.64$; with the solar CZ proper being thought to occupy the region $r \gtrsim 0.7$, the region $r_{0} \leq r \lesssim 0.7$ can be thought of as an overshoot region/tachocline, where the major radial gradients of $\Omega$ occur. Equations (1) and (2) were solved using a modified version of the code described in Moss $\&$ Brooke (2000), using the above boundary conditions, over the range $r_{0} \leq r \leq 1,0 \leq \theta \leq \pi$. We used a mesh resolution of $61 \times 101$ points, uniformly distributed in radius and latitude respectively, i.e. $\Delta r=0.06, \Delta \theta=0.01 \pi$.

\subsection{Density stratification}

The density profile in the solar model computed by Christensen-Dalsgaard et al. (1996; henceforth referred to as CD96) is shown in Fig. 1. We approximated the density stratification in the solar convection zone by a polytropic density profile, writing in general

$\rho(r) / \rho_{0}=\left(\frac{r_{s}-r}{r_{s}-r_{0}}\right)^{n}$

where

$r_{s}=\left(\frac{\rho_{\text {ratio }}^{1 / n}-r_{0}}{\rho_{\text {ratio }}^{1 / n}-1}\right)$

we take $n=1.5$ below. This density profile corresponds to a polytrope truncated at the appropriate radius to give the required value of $\rho_{\text {ratio }}$, with this radius subsequently renormalized to unity. $\rho_{\text {ratio }}$ is thus the ratio of the densities at the top 


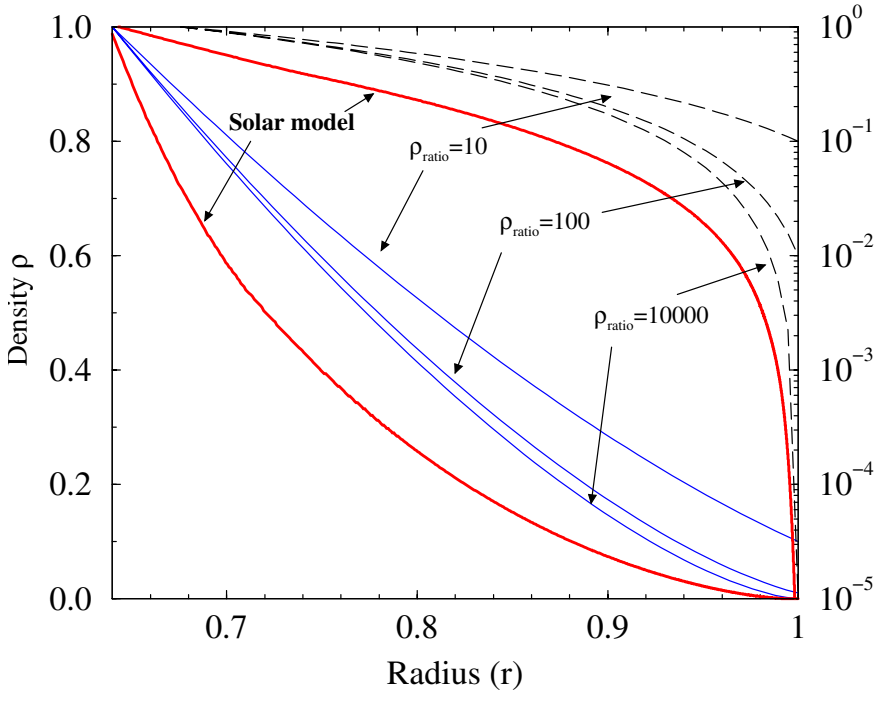

Fig. 1. Density profiles obtained using a polytropic model, for several values of $\rho_{\text {ratio }}$. Here $\rho$ is dimensionless, measured in units of the density at $r=r_{0}$. The upper set of curves show $\log \rho$, the lower show $\rho$. Shown also is the density profile of CD96. For $\rho_{\text {ratio }} \gtrsim 10^{2}$, the density profile is approximately invariant through most of convection zone, apart from the regions near the surface.

$(r=1)$ and the bottom $\left(r=r_{0}\right)$ of the computational domain; $\rho_{0}=\rho\left(r_{0}\right)$. Several examples of such profiles, with different values of $\rho_{\text {ratio }}$ are also shown in Fig. 1 . For $\rho_{\text {ratio }} \gtrsim 100$, these give a reasonable approximation to the density stratification in the bulk of the solar CZ, apart from the outermost regions near the solar surface

\subsection{Explicit $\alpha$-quenching}

The nonlinearity in our original model is through the nonlinearity in the Lorentz force and the subsequent presence of the $v^{\prime}$ term in the induction equation. In all of our previous studies the form of $\alpha_{\mathrm{r}}$ was chosen to be independent of the magnetic field $\boldsymbol{B}$. It did, however, include an implicit $\alpha$-quenching in the overshoot layer in that $\alpha$ was prescribed to vanish in $r \leq 0.7$ and to be relatively small in the layers immediately above where $|\boldsymbol{B}|$ was anticipated to be relatively large. Here we study the effects of having an additional nonlinearity in the form of an explicit nonlinear $\alpha$-quenching given (reverting temporarily to dimensional units) by

$\alpha=\alpha_{\mathrm{r}}(r) \frac{\sin ^{2} \theta \cos \theta}{1+g^{\prime}\left|\boldsymbol{B}^{2}\right| / B_{\mathrm{eq}}^{2}}$

where $B_{\text {eq }}=\left(4 \pi \rho v_{t}^{2}\right)^{1 / 2}$ is the equipartition field strength, $v_{t}$ is the turbulent velocity and $g^{\prime}$ is of order unity. (The trigonometric factor is chosen to agree with that used in our earlier work - see above.) In order to estimate how $B_{\text {eq }}^{2}$, appearing in the denominator, varies with depth we recall that to a very good approximation the luminosity $L=4 \pi r^{2} F_{r} \propto r^{2} \rho v_{t}^{3}$ is a constant in the CZ, which gives $v_{t} \propto \rho^{-1 / 3} r^{-2 / 3}$ and hence $\rho v_{t}^{2} \propto \rho^{1 / 3} r^{-4 / 3}$. This suggests that the denominator in Eq. (5) will be a slowly varying function of the depth, especially away from the solar surface, and this expectation is supported by detailed mixing length models of the solar CZ. Thus we take $B_{\text {eq }}^{2} \propto \rho v_{t}^{2}=$ const, and so the $\alpha$-quenching expression (5) can be well approximated (now temporarily using dimensionless variables again) by

$\tilde{\alpha}=\alpha_{\mathrm{r}}(r) \frac{\sin ^{2} \theta \cos \theta}{1+g\left|\boldsymbol{B}^{2}\right|}$,

where $g=g^{\prime} \Lambda 4 \pi \rho_{0} \eta_{0} \Omega^{*} / B_{\text {eq }}^{2} . B_{\text {eq }}^{2}$ is constant, and thus so is $g$. (In this connection it may be noted that there is an ongoing controversy regarding the nature and strength of $\alpha$-quenching. This is not the place to rehearse the arguments for and against 'strong' alpha-quenching (where $g^{\prime} \gg 1$ ); the issue is unresolved, and we use Eq. (6) as a commonly adopted nonlinearity.)

We can make an estimate of what might be plausible values for $g$ by taking $\eta_{0}=3 \times 10^{11} \mathrm{~cm}^{2} \mathrm{~s}^{-1}$ (see Sect. 3 below), $\Lambda=10^{4}, \rho_{0}=1 \mathrm{gm} \mathrm{cm}^{-3}, \Omega^{*}=3 \times 10^{-6} \mathrm{~s}^{-1}$, $B_{\text {eq }}=3500 \mathrm{G}$ (the latter estimated from an envelope model by Baker \& Temesvary (1966) (more recent models do not give significantly different values). Then $g \sim 3 \times 10^{3} g^{\prime}$. (As noted above, $B_{0}^{2}, g \propto \Lambda$, but $\tilde{\boldsymbol{B}} \propto \Lambda^{-1 / 2}$, so the results in physical variables do not depend on the value adopted for $\Lambda$.)

The inclusion of both types of nonlinearity in the model allows us to study the relative importance of each term and in particular what happens to the amplitudes of the torsional oscillations as $g$ increases.

\section{Results: Effects of density stratification}

Using the above model, we studied the dynamics in the convection zone, and in particular the changes caused by the introduction of the density stratification and the additional nonlinearity. We concentrated on the effects of these changes on the torsional oscillations and in particular on their amplitudes (i.e. the angular velocity residuals, defined as the current angular velocity minus the background angular velocity $\Omega_{0}$ ), since these are the most important indicators to be compared with observations. The model predictions are likely to be of particular importance in deeper regions where the uncertainties in the inversions are largest.

We first study the effects of density stratification in isolation, with $g=0$. We fixed $R_{\omega}=6 \times 10^{4}$ (corresponding to $\eta_{0} \approx 3 \times 10^{11} \mathrm{~cm}^{2} \mathrm{~s}^{-1}$ ) by requiring that the dynamo period is near to 22 years close to marginal stability. The marginal value of $R_{\alpha}$ is then about -3.2 .

\subsection{Comparison with previous results}

To begin with we made a detailed comparison of the results obtained here in the presence of density stratification, with our previous studies which ignored this effect. In particular, we concentrated on one of the findings of these earlier studies, namely the fact that torsional oscillations were found to extend all the way down to the bottom of the $\mathrm{CZ}$.

Our results here show that, for both near-critical, and moderately supercritical dynamo regimes, the oscillations extend all the way down to the bottom of the convection zone, for all 


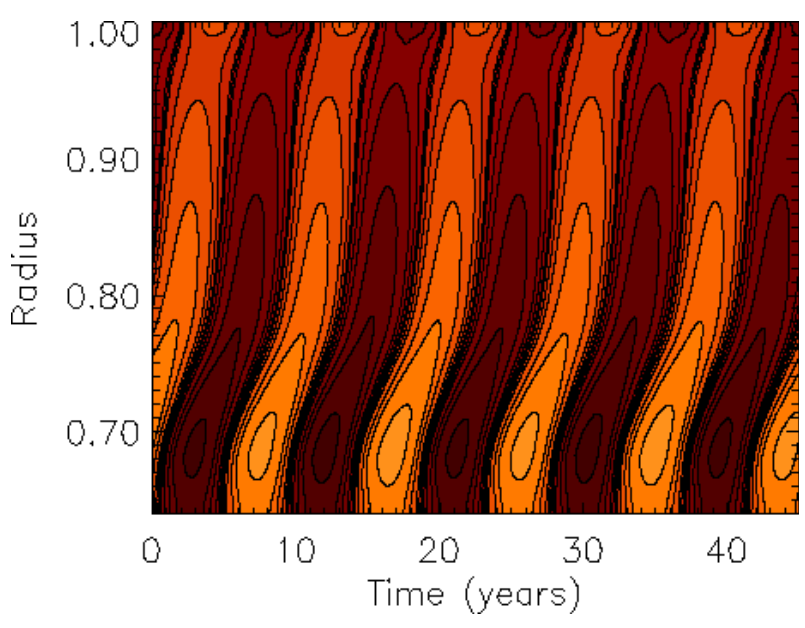

Fig. 2. The radial $(r-t)$ contours of the angular velocity residuals $\delta \Omega$ as a function of time for a cut at 10 degrees latitude. The model parameters are $R_{\alpha}=-4.0, P_{\mathrm{r}}=1.0, R_{\omega}=60000$ and $\rho_{\text {ratio }}=10000$. Note the penetration of the torsional oscillations all the way down to the bottom of the convection zone. Darker and lighter regions represent positive and negative deviations from the time averaged background rotation rate.

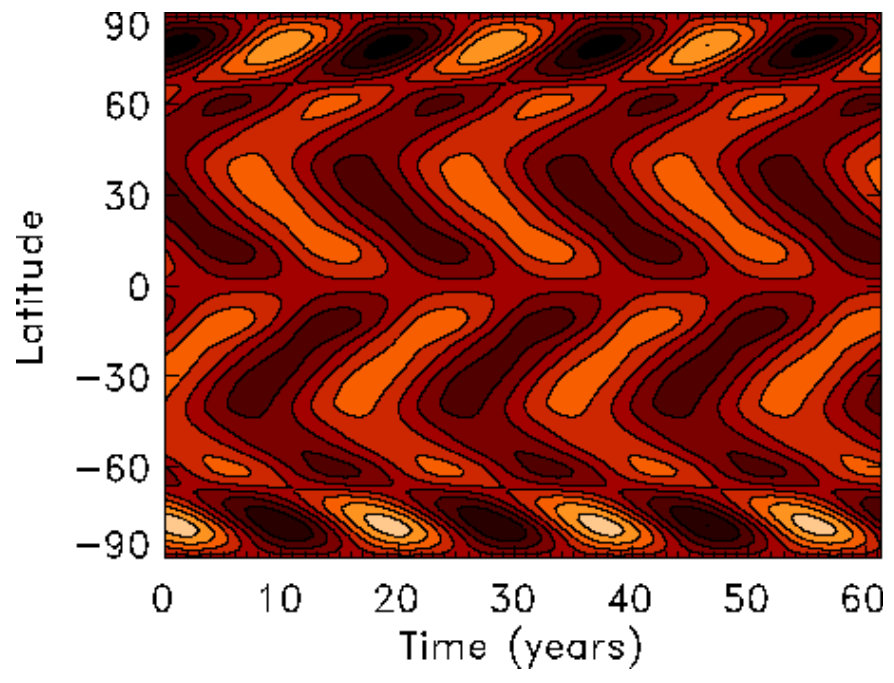

Fig. 3. Butterfly diagram of the toroidal component of the magnetic field $\boldsymbol{B}$ at $r=0.99$. The parameters are the same as those for Fig. 2. Dark and light shades correspond to positive and negative values of $B_{\phi}$ respectively.

$1 \leq \rho_{\text {ratio }} \leq 10^{6}$. This is in line with our previous results which shows that such behaviour is an extremely robust feature of the models considered to density stratification. An example of such generic penetration is depicted in Fig. 2. This is important observationally since the inversions have so far not been able to resolve unambiguously regimes near the bottom of the convection zone (Howe et al. 2000a; Antia \& Basu 2000).

For completeness we also show in Fig. 3 the butterfly diagram (i.e. latitude-time plot) for the toroidal component of the magnetic field $\boldsymbol{B}$ near the surface, as well as the corresponding plot for the angular velocity residuals (i.e. the near-surface torsional oscillations) in Fig. 4. As can be seen, the presence of significant density stratification does not alter the qualitative behaviour of the magnetic field and torsional oscillations.

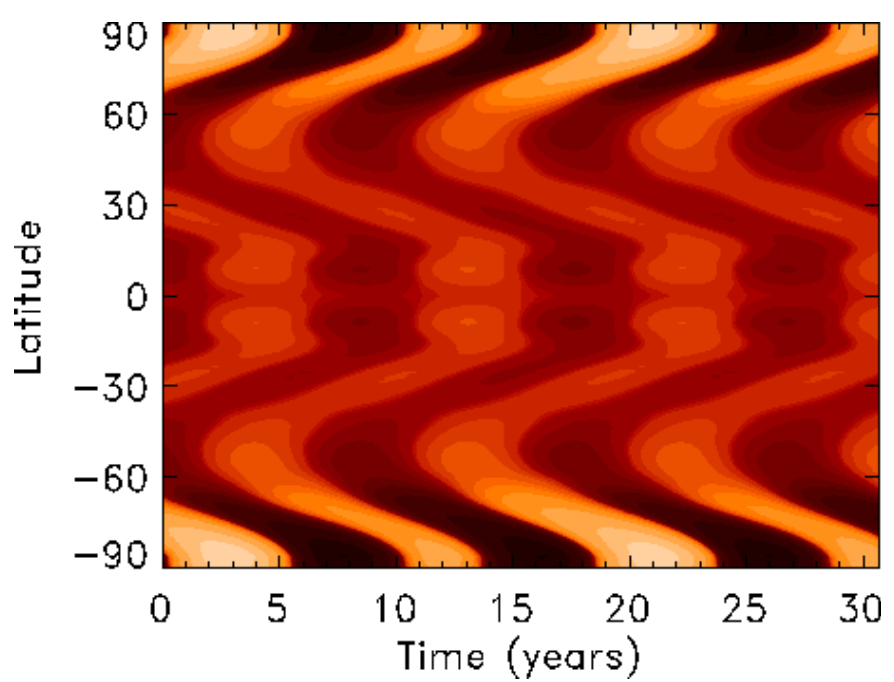

Fig. 4. Angular velocity residuals at $r=0.99$ with latitude and time. The parameters are the same as those for Fig. 2. A temporal average has been subtracted to reveal the migrating banded zonal flows. Darker and lighter regions represent positive and negative deviations from the time averaged background rotation rate.

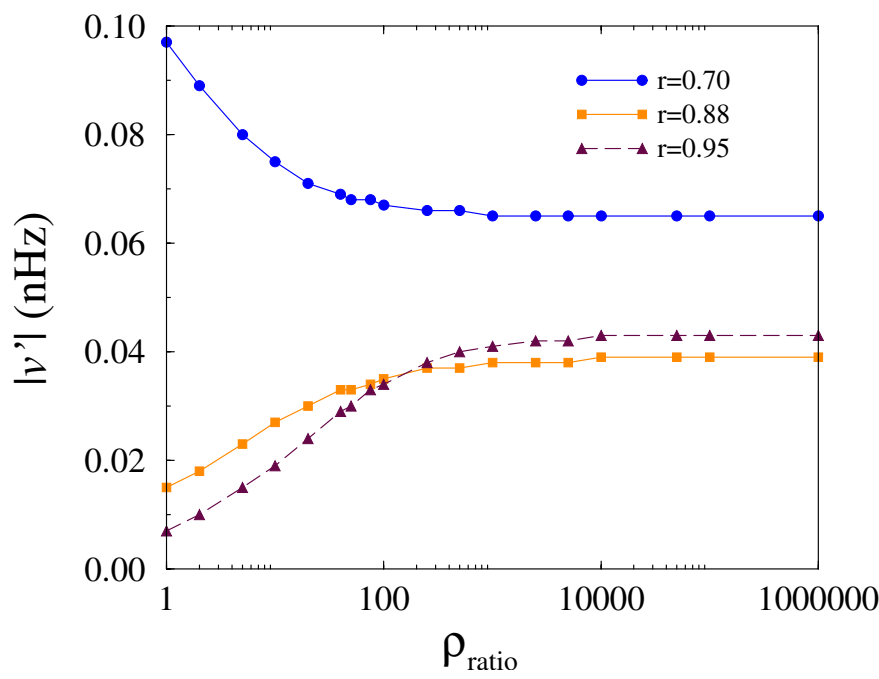

Fig. 5. The variation of oscillation amplitudes at $r=0.70,0.88$ and 0.95 as a function of $\rho_{\text {ratio }}$, for a near-onset dynamo regime. The parameters are $R_{\alpha}=-3.2, P_{\mathrm{r}}=1.0, R_{\omega}=60000$, with $\alpha=\alpha_{r}(r) f(\theta)$ and no $\alpha$-quenching.

\subsection{Amplitudes of oscillations as a function of $\rho_{\text {ratio }}$}

From an observational point of view, an important feature of the dynamics of the $\mathrm{CZ}$ is the way the amplitudes of the torsional oscillations vary as a function of model ingredients and parameters, as well as depth in the CZ. A theoretical understanding is crucially important here, especially given the uncertainties in inversions of the helioseismic data in the lower parts of the CZ.

With this in mind, we studied how the amplitudes of the torsional oscillations change as a function of $\rho_{\text {ratio }}$, for two values of $R_{\alpha}$; one corresponding to a near-critical and the other to a supercritical dynamo regime. The results are depicted in Figs. 5 and 6. For orientation, we recall that the corresponding 




Fig. 6. The variation of oscillation amplitudes at $r=0.70,0.88$ and 0.95 as a function of $\rho_{\text {ratio }}$, for a supercritical case. The parameters are $R_{\alpha}=-4.4, P_{\mathrm{r}}=1.0, R_{\omega}=60000$, with $\alpha=\alpha_{r}(r) f(\theta)$ and no $\alpha$-quenching.

amplitudes in the sun, deduced from helioseismological observations, are of the order of one $\mathrm{nHz}$.

The important trend revealed by these figures is that, in both the near-critical and supercritical cases, the amplitudes of the torsional oscillations in the upper part of the $\mathrm{CZ}$ increase as $\rho_{\text {ratio }}$ increases, while those at the bottom decrease. The decrease in amplitude of the torsional oscillations in the bottom of the convection zone, as $\rho_{\text {ratio }}$ increases (i.e. as the density profile becomes more realistic) is of practical importance, as it suggests that the detection of such oscillations may be even more difficult than might appear at present just from the limits to the resolution of the helioseismological inversions at the bottom of the convection zone. The increase of the amplitudes at the top is also important since it improves the agreement between simulated and observed values.

Also of potential interest is the way that the distribution of the perturbation kinetic energy, defined by $\int_{V} \rho\left(2 v_{0} v^{\prime}+v^{\prime 2}\right) \mathrm{d} V$, is modified as the density stratification changes. In Fig. 7 we show the variation of this integral with radius, where we take the domain $V$ of integration to be the local spherical shell with thickness $\Delta r$. Introducing a stratification markedly reduces the perturbation kinetic energy in the near-surface regions, but the differences between the two stratified models illustrated are relatively small. However the low values of the near-surface density in the stratified models means that the amplitude of the torsional oscillations in this region are not correspondingly decreased - see Figs. 5 and 6 . This relative insensitivity of the observed quantity to the stratification is reassuring.

\subsection{Amplitudes of oscillations as a function of $R_{\alpha}$}

To begin with, we verified that for a given density profile (in presence of density stratification), the amplitudes of the oscillations increase as the dynamo number $R_{\alpha}$ is increased (see also Covas et al 2001a). The results are shown in Fig. 8. We then studied how the amplitudes change as we change the density

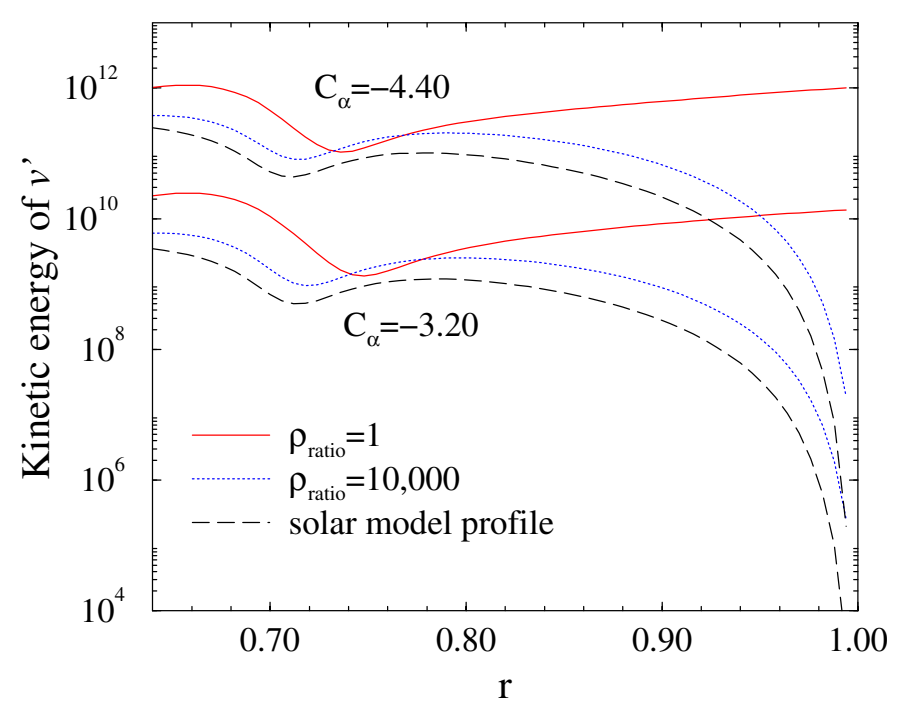

Fig. 7. The distribution of the kinetic energy (in arbitrary units) in spherical shells of thickness the radial mesh size $\Delta r$, as a function of radius and $\rho_{\text {ratio }}$, for both near-onset and supercritical dynamo regimes. The parameters are as in Figs. 5 and 6 respectively.



Fig. 8. The variation of oscillation amplitudes as a function of $R_{\alpha}$. Parameter values are $P_{\mathrm{r}}=1.0$ and $R_{\omega}=60000, \rho_{\text {ratio }}=10000$, with $\alpha=\alpha_{r}(r) f(\theta)$ and no $\alpha$-quenching.

profile by increasing the value of $\rho_{\text {ratio }}$. For comparison we also calculated the amplitudes for the density profile of CD96. The results are shown in Fig. 9. As can be seen, the effect of increasing $\rho_{\text {ratio }}$ is to increase the amplitudes of oscillations near the surface. The largest amplitudes are attained for the CD96 density distribution.

\subsection{Amplitudes of oscillations as a function of depth and $\rho_{\text {ratio }}$}

We also studied the variations of the amplitudes of oscillations as a function of depth for a number of values of $\rho_{\text {ratio }}$, for both a near-onset regime (with $R_{\alpha}=-3.2$ ) as well as for more supercritical parameters $\left(R_{\alpha}=-4.4\right)$, and the results are shown in Figs. 10 and 11 respectively. As can be seen, despite the latter 


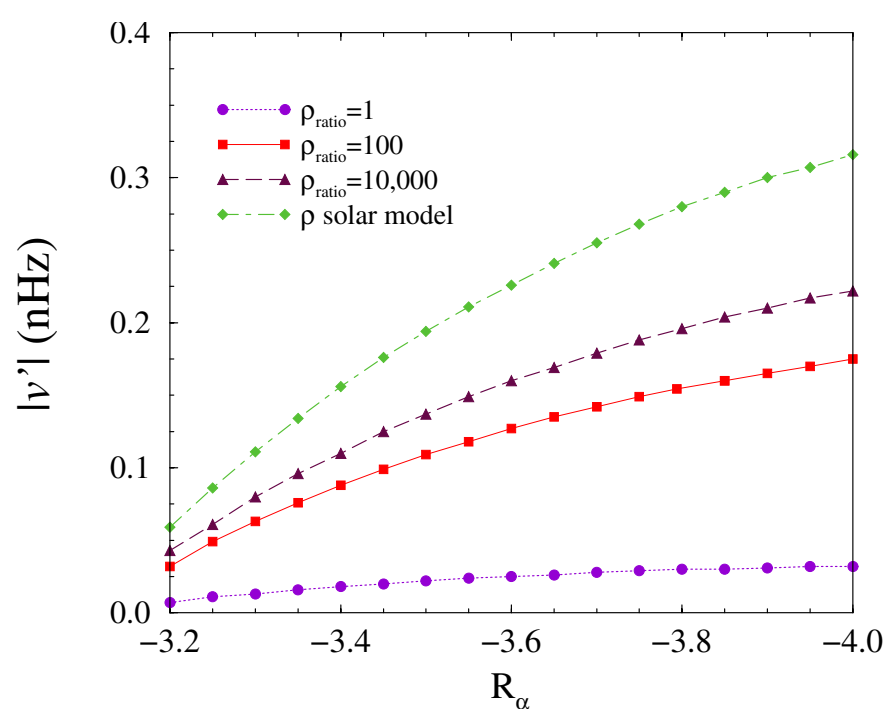

Fig. 9. The variation of oscillation amplitudes as a function of $R_{\alpha}$ for different values of $\rho_{\text {ratio }}$ at $r=0.95$. Shown also are the variations obtained using the solar model density profile of CD96. The parameters are $R_{\omega}=60000, P_{\mathrm{r}}=1.0$ with $\alpha=\alpha_{r}(r) f(\theta)$ and no $\alpha$-quenching.

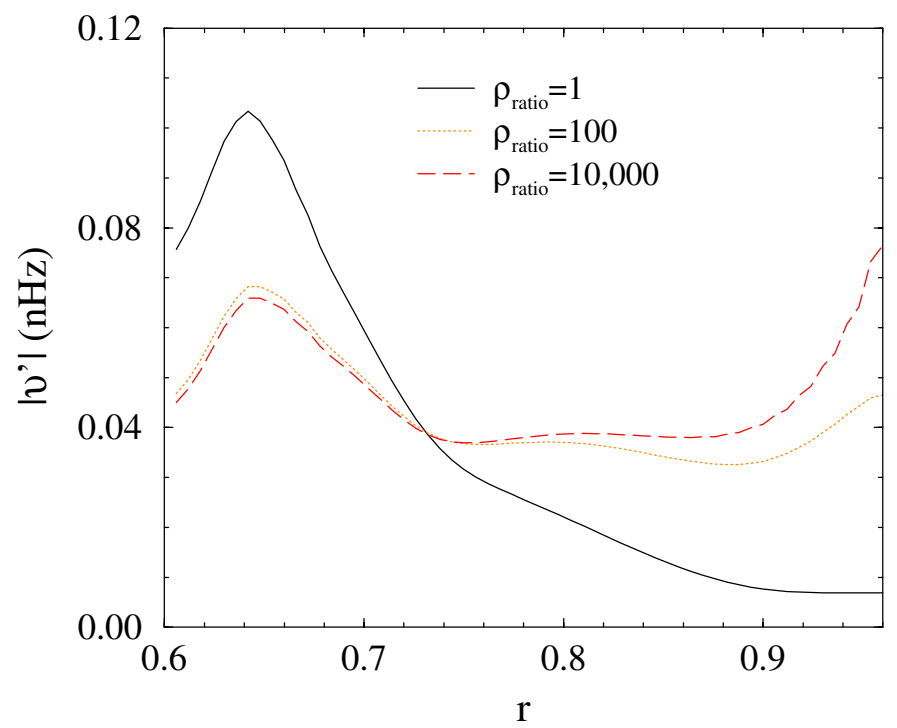

Fig. 10. The variation of oscillation amplitudes as a function of depth for a number of values of $\rho_{\text {ratio }}$, for a near-onset regime with $R_{\alpha}=$ $-3.2, R_{\omega}=60000, P_{\mathrm{r}}=1.0, \alpha=\alpha_{r}(r) f(\theta)$ and no $\alpha$-quenching.

having larger amplitudes, the two cases have important similarities. In each the amplitudes near the surface layers increase with increasing $\rho_{\text {ratio, }}$, whereas they decrease near the bottom.

\section{Results: Comparative study of the nonlinearities}

In order to make a comparative study of the effects of the two competing forms of nonlinearities considered here, we begin by recalling that, in the absence of any alpha-quenching, increasing the degree of nonlinearity due to the Lorentz force (i.e. increasing $R_{\alpha}$ ) results in an increase in the amplitudes of the torsional oscillations (see also below). The question is what is the effect of increasing the strength of the additional nonlinearity due to $\alpha$-quenching on these amplitudes?

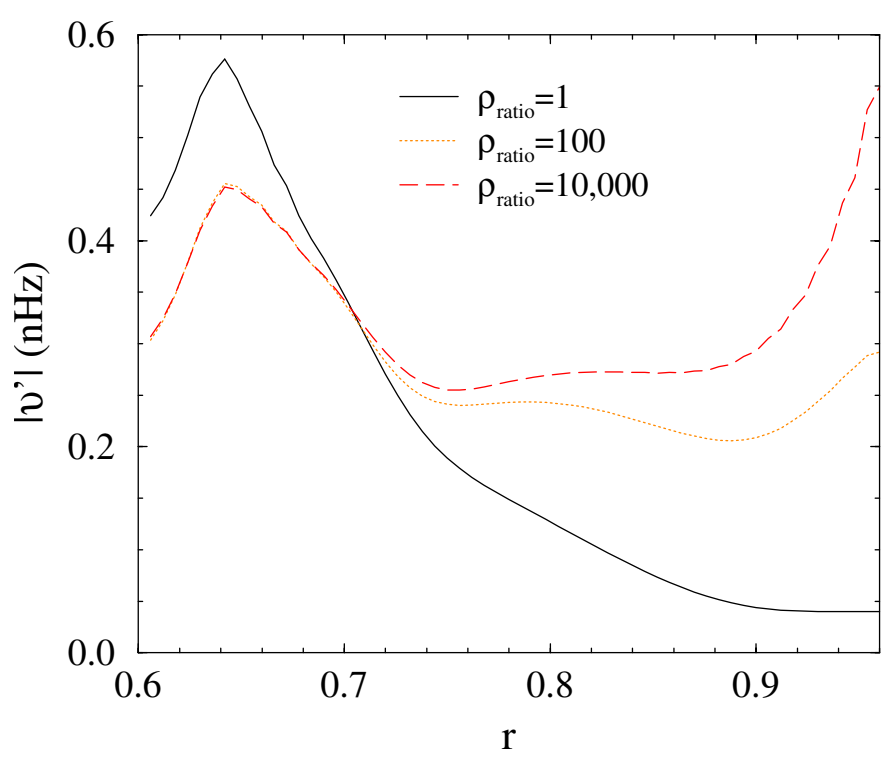

Fig. 11. The variation of oscillation amplitudes as a function of depth for a number of values of $\rho_{\text {ratio }}$, for a supercritical case with $R_{\alpha}=-4.4$, $R_{\omega}=60000, P_{\mathrm{r}}=1.0, \alpha=\alpha_{r}(r) f(\theta)$ and no $\alpha$-quenching.

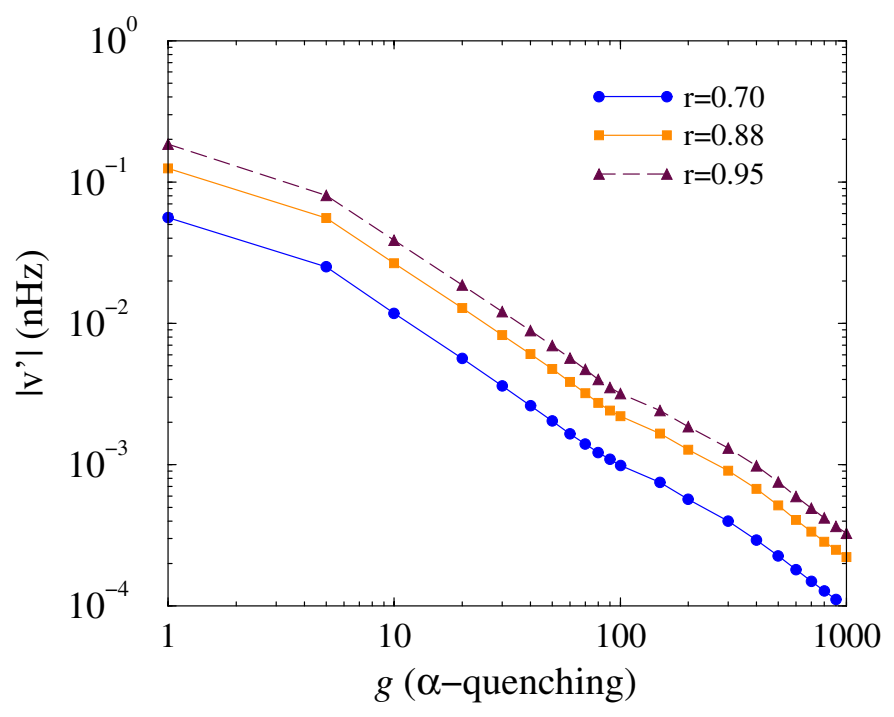

Fig. 12. The variation of amplitudes of torsional oscillations as a function of changes in the $\alpha$-quenching coefficient $g$ for a near-onset regime. Parameters are $R_{\alpha}=-2.5, P_{\mathrm{r}}=1.0, R_{\omega}=60000$ with $\alpha_{\mathrm{r}}=1$ (i.e. no explicit radial dependence of $\alpha$ ) and the density $\rho$ given by the solar model of CD96.

We studied this question by making a detailed study of how the amplitudes of torsional oscillations change as a function of $g$, the coefficient of the $\alpha$-quenching term (Eq. (5)). The results are presented in Fig. 12. A number of trends are apparent. The amplitudes of the torsional oscillations decrease at all depths with increasing $\alpha$-quenching coefficent $g$. However, the oscillations at the top of the $\mathrm{CZ}$ have the highest amplitudes throughout.

To study the influence of increasing the $\alpha$-quenching coefficient, we recall that in the case of pure alpha-quenching,

$\left\langle\boldsymbol{B}^{2}\right\rangle \propto \frac{1}{g}$, 


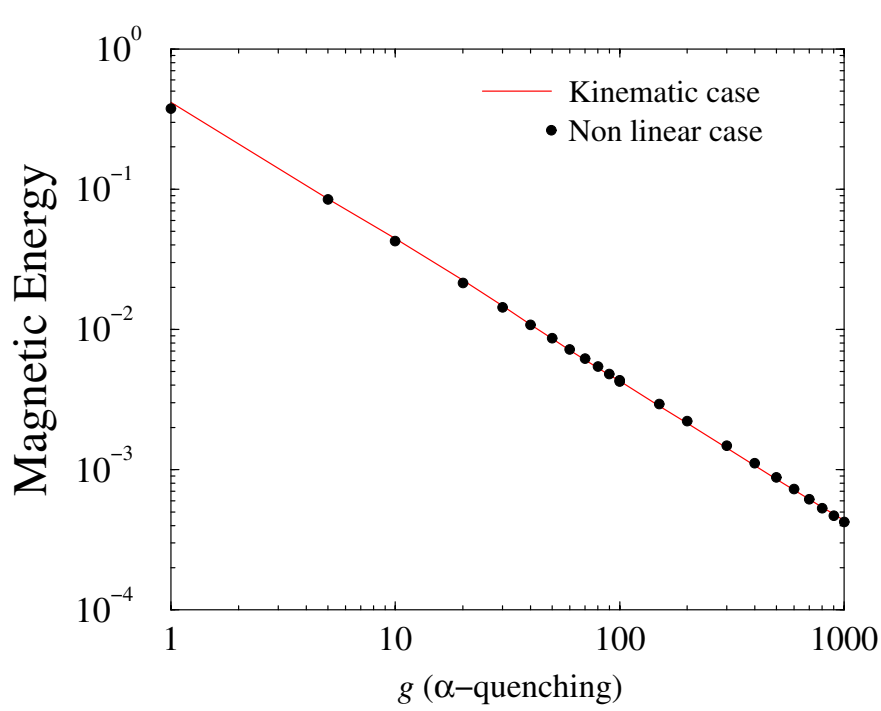

Fig. 13. The variation of the magnetic energy as a function of the $\alpha$-quenching coefficient $g$. Shown are both the kinematic (without the Navier-Stokes equation) and the nonlinear (with the Navier-Stokes equation) cases. Parameters are $R_{\alpha}=-2.5, P_{\mathrm{r}}=1.0$ and $R_{\omega}=60000$, with $\alpha_{\mathrm{r}}=1$ and the density $\rho$ given by the solar model of CD96.

where the angled parentheses denote some sort of global average. We checked that this was exactly satisfied for our code in the quasi-kinematic approximation when the Navier-Stokes equation was switched off. Interestingly, we also found similar behaviour when the additional nonlinear feedback via the Navier-Stokes equation was present. This is shown in Fig. 13, which displays the behaviour of the global magnetic energy as a function of $g$.

The amplitudes of the torsional oscillations increase somewhat with $\left|R_{\alpha}\right|$ but in the range accessible to our code $\left(\left|R_{\alpha}\right| \lesssim\right.$ $15)$ this increase is insufficient to compensate for the decrease caused by the increase in the $\alpha$-quenching coefficient $g$ (given by Eq. (7)).

We can add here that in our previous, very preliminary, study of the effects of alpha-quenching on the torsional oscillations (Covas et al 2001a), we were only able to find oscillatory solutions for very small values of the alpha-quenching coefficient $(g<0.1)$. What has enabled us to find the existence of oscillatory solutions at such large values of $g$ here is our recent discovery of the existence of multiple attractors in the dynamo system, including both oscillatory and steady solutions, that coexist to $g \sim 1000$, i.e. in the physically plausible range as estimated in Sect. 2.3.

\section{Discussion}

We have made a detailed study of the effects of including density stratification in the model, as well as an additional form of nonlinearity due to $\alpha$-quenching on the behaviour of solar torsional oscillations. We feel that the previous omission of any discussion of these effects reduced the detailed applicability of our results to the observational data.

To study the effects of density stratification, first of all we made a comparison with our previous results and found both important similarities and differences. Concerning similarities, we found that consistent with our previous findings for nearcritical and moderately supercritical dynamo regimes, the torsional oscillations extend all the way down to the bottom of the convection zone. This is true in presence of density stratification with all values of $\rho_{\text {ratio }}$ in the range $\left[1-10^{6}\right]$. Concerning differences, we found that in both the near-critical and supercritical cases, the amplitudes of the torsional oscillations in the upper part of the $\mathrm{CZ}$ increase with $\rho_{\text {ratio }}$, while those at the bottom decrease. Given that the uncertainties in the inversions are expected to be greatest at the bottom of the $\mathrm{CZ}$, the lowering of amplitudes in these regions with increasing density stratification has potential importance for the analysis of helioseismological data, and for the understanding of the dynamo regimes near the bottom of the convection zone.

When two forms of nonlinearity are simultaneously present, we find that torsional oscillations persist in presence of both nonlinearities, for a large range of values of the $\alpha-$ quenching coefficient $g$ up to $g>1000$. The amplitudes of these oscillations decrease however, as they shadow the square of the magnetic field strength, which in turn follows the scaling with $g$ given by Eq. (7).

We also note that for small $g$, the combined effect of increasing $\rho_{\text {ratio }}$ and $g$ is to leave the amplitude of the nearsurface torsional oscillations little changed. However, for larger $g(\gtrsim 10)$, the effect of $\alpha$-quenching dominates, as the amplitudes of the torsional oscillations scale as $1 / \mathrm{g}$.

Equation (2) suggests, and our models confirm, that variations in $v^{\prime}$ closely shadow those in the $\phi$-component of the Lorentz force. It seems a priori difficult to imagine a driver for torsional oscillations that does not involve the Lorentz force, either at a macro- or micro-level (e.g. the "nonlinear $\Lambda$-effect", Kitchatinov \& Pipin 1998; Kitchatinov et al. 1999), whatever the prime nonlinearity in the dynamo. Thus the requirement that the Lorentz force shadows the torsional oscillations is plausibly an additional constraint even on dynamo models that do not explicitly include dynamical effects (e.g. conventional $\alpha$ quenched models. Brandenburg \& Tuominen 1988 appear to have been the first to investigate semi-quantitatively such an idea.). However it is perhaps a little puzzling that values of $g$ as large as suggested by the estimates of Sect. 2.3 reduce the amplitudes of the torsional oscillations so substantially, removing the previous agreement with observations.

\section{References}

Antia, H. M., \& Basu, S. 2000, ApJ, 541, 442

Baker, N. H., \& Temesvary, S. 1966, Tables of Convective Stellar Envelopes (NASA)

Brandenburg, A., \& Tuominen, I. 1988, Adv., Space Res., 8, 185

Christensen-Dalsgaard, J., Däppen, W., Ajukov, S. V., et al. 1996, Science, 272, 1286

Covas, E., Tavakol, R., Moss, D., \& Tworkowski, A. 2000a, A\&A, 360, L21

Covas, E., Tavakol, R., \& Moss, D. 2000b, A\&A, 363, L13

Covas, E., Tavakol, R., \& Moss, D. 2001a, A\&A, 371, 718

Covas, E., Tavakol, R., Vorontsov, S., \& Moss, D. 2001b, A\&A, 375, 260

Covas, E., Moss, D., \& Tavakol R. 2002, A\&A, 394, 1117 (Erratum)

Howard, R., \& LaBonte, B. J. 1980, ApJ, 239, L33 
Howe, R., Christensen-Dalsgaard, J., Hill, F., et al. 2000a, ApJ, 533, L163

Howe, R., Christensen-Dalsgaard, J., Hill, F., et al. 2000b, Science 287,2456

Kitchatinov, L. L., \& Pipin, V. V. 1998, Astron. Rep., 42, 808

Kitchatinov, L. L., Pipin, V. V., Makarov, V. I., \& Tlatov, A. G. 1999, Sol. Phys., 189, 227

Kitchatinov, L. L., Mazur, M. V., \& Jardine, M. 2000, A\&A, 359, 531

Kosovichev, A. G., \& Schou, J. 1997, ApJ, 482, 207

Moss, D., Mestel, L., \& Tayler, R.J. 1990, MNRAS, 245, 550

Moss, D., \& Brooke, J. 2000, MNRAS, 315, 521

Rüdiger, R., \& Brandenburg, A. 1995, A\&A, 296, 557

Schou, J., Antia, H. M., Basu, S., et al. 1998, ApJ, 505, 390

Snodgrass, H. B., Howard, R. F., \& Webster, L. 1985, Sol. Phys., 95, 221
Tavakol, R., Covas, E., Moss, D., \& Tworkowski, A. 2002, A\&A, 387, 1100

Vorontsov, S. V., Christensen-Dalsgaard, J., Schou, J., Strakhov, V. N., \& Thompson, M. J. 2002a, Science, 296, 101

Vorontsov, S. V., Tavakol, R., Covas, E., \& Moss, D. 2002b, The Evolving Sun and Its Influence on Planetary Environments, ed. B. Montesinos, A. Gimenez, \& E. F. Guinan, ASP Conf. Ser., 269, 233

Vorontsov, S. V., Covas, E., Moss, D., \& Tavakol, R. 2003, Solar cycle variation of the solar internal rotation: helioseismic measurements versus dynamo modelling Local and global helioseismology: the present and future, ed. H. Sawaya-Lacoste, ESA SP-517, The Netherlands: ESA Publications Division, ISBN 929092-827-1, 35 